\title{
Isolation of neuronal cells with high processing activity for the Machado-Joseph disease protein
}

\author{
Dear Editor,
}

Protein processing is increasingly being recognized to be extremely important in a wide variety of cell functions, not only in physiological conditions, but also in many pathological conditions, especially in several neurodegenerative disorders. ${ }^{1}$ However, such processing activities are usually very weak and very difficult to analyze. Here we show the 'cleavage-dependent cell selection system' in which a weak protein processing activity is converted into a strong transcriptional activity, thus allowing the expression of drug resistance proteins required for selection. This system will provide a novel cell selection system for isolating cells containing processing activities for any protein concerned, and will contribute to the identification of yet unknown processing enzymes involved in various cell functions.

A class of inherited human neurodegenerative disorders, including Huntington disease (HD), Machado-Joseph disease (MJD), and spinobulbar muscular atrophy (SBMA) has been found to be caused by expanded CAG repeats encoding polyglutamines, ${ }^{1-3}$ and is thus called 'the polyglutamine diseases'. ${ }^{4,5}$ Although the gene responsible for each disease is ubiquitously expressed in most tissues as well as throughout the nervous system, each disorder has its own susceptible regions in the central nervous system. ${ }^{4-6}$ We previously isolated the MJD gene, ${ }^{2}$ the gene responsible for MJD, and demonstrated that expanded polyglutamines, if expressed as part of a fragment of the MJD protein, but not when expressed as part of the entire MJD protein, aggregate and trigger cell death in cultured cells, and induce neurodegeneration in vivo in transgenic mice. ${ }^{7}$ From these results, we have proposed the 'processing model', in which cleavage or processing of the diseased protein is a crucial step in the pathogenesis of the polyglutamine diseases; ${ }^{4,5,7}$ namely, the brain regionspecific cleavage of the diseased proteins with expanded polyglutamines leads to the degeneration of specific brain regions in each disease. Several lines of evidence supporting this model have been demonstrated. ${ }^{8-12}$

In order to find further evidence to substantiate the 'processing model', we attempted to identify cultured cells possessing MJD protein processing activity. For this purpose, we expressed MJD79 in a variety of cell lines, and immunocytochemically examined the MJD79 protein expressed. ${ }^{2,7,13}$ Unfortunately, we were initially unable to find any evidence suggesting cleavage of the MJD protein in any of the cultured cells examined, which were all dividing cells. However, when neuronally differentiated post mitotic PC12 cells were examined several days after transfection of MJD79, we noticed that approximately one in a few hundred of the transfected PC12 cells made polyglutamine-mediated aggregates in the cells; these aggregates could be detected only with an antibody which recognizes an amino acid stretch just downstream of the polyglutamine portion in the MJD protein, but not with an antibody to a tag attached to the N-terminus of the MJD protein. ${ }^{13}$ With a similar frequency, aggregate formation was detected in living cells upon transfection of MJD79GFP, but not GFP-MJD79, again only in differentiated PC12 cells several days after transfection (Figure 1A). These aggregates observed within the homogenous GFP signals were indistinguishable from those observed upon Q79-GFP transfection. These observations suggested that a very small population of PC12 cells is capable of cleaving the MJD protein, and due to the fact that this activity is very weak, the cleaved MJD product does not accumulate enough to form aggregates in dividing cells.

We have previously established a system for the enhancement of a weak transcriptional activity by greater than a 100 -fold. ${ }^{14}$ Modifying this system, we designed the 'cleavage-dependent cell selection system', in which a very weak protein processing activity can theoretically be converted into a strong transcriptional activity. For example (Figure 1B), the full-length MJD protein with Gal4VP16 (an artificial strong transcriptional activator) ${ }^{14}$ fused to its Cterminus, is expressed together with a Gal4 reporter plasmid $^{14}$ which drives the expression of a drug resistant marker protein, in this case for Zeocin resistance. More specifically, the system should work in the following way: when the MJD protein, MJD35 for example, is cleaved by its processing enzyme, the cleaved portion with Gal4VP16 will translocate to the nucleus and transactivate the reporter gene, thereby resulting in Zeocin resistance in dividing predifferentiated PC12 cells. The Zeocin-resistant cells obtained from this system should contain PC12 subclones with enhanced processing activity of the MJD protein. By repeating this selection process, cells with a much higher processing activity for the MJD protein should be obtained. We used MJD35, which contains a normal length of polyglutamine repeats in the selection process, in order to avoid aggregate formation of the cleaved fragments which should result in cell death. In order to exclude the noncleaved MJD35-Gal4VP16 protein from the nucleus, the transmembrane domain of the Fas receptor ${ }^{15}$ was fused to the N-terminus of the MJD35-Gal4VP16 protein. The TMMJD35-Gal4VP16 protein was confirmed to be exclusively attached to the cell membrane by immunocytochemical analysis (data not shown).

We were indeed able to obtain several PC12 subclones after selection with $100 \mu \mathrm{g} / \mathrm{ml}$ Zeocin (Figure 1C, a). Among 
A
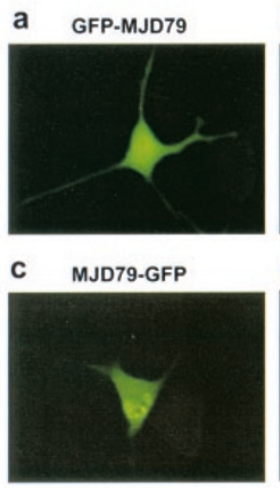

b

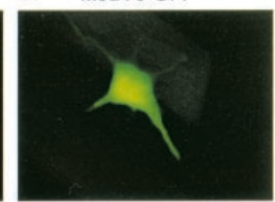

d

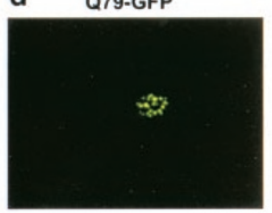

C

a

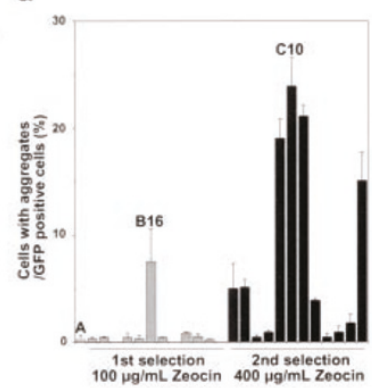

b

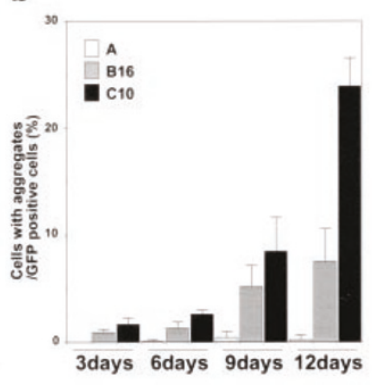

B

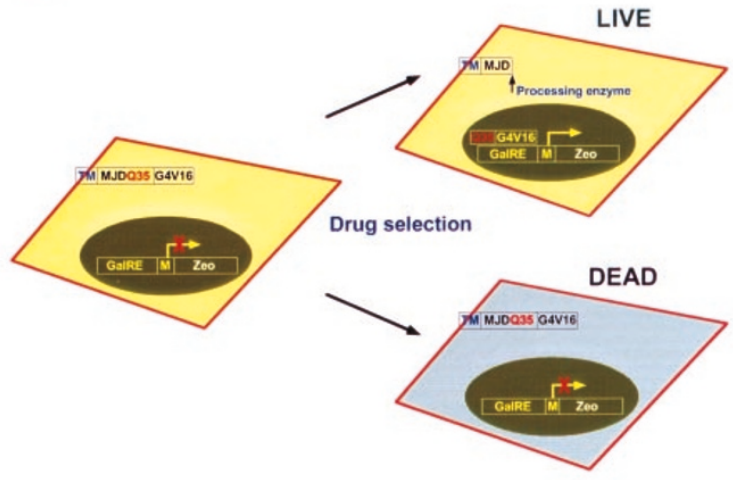

D

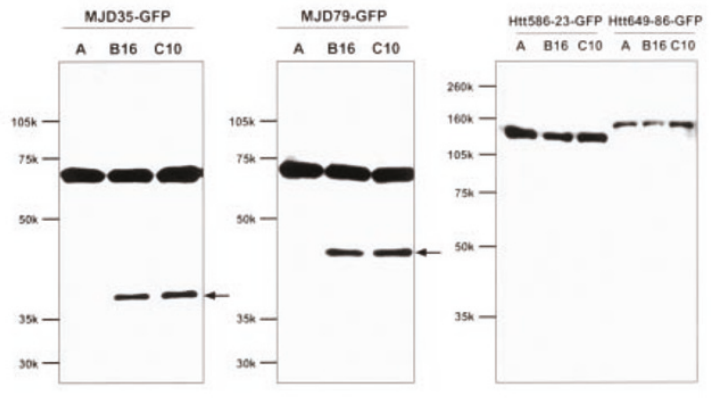

Figure 1 (A) Visualization of polyglutamine-mediated aggregates in living cells. PC12 cells were transfected with an expression vector for GFP-MJD79 (an Nterminally GFP-tagged full-length MJD protein containing a pathological length of 79 polyglutamine repeats), MJD79-GFP (a C-terminally GFP-tagged MJD79), or Q79-GFP (a C-terminally GFP-tagged 79 polyglutamine stretch from the MJD protein), and the proteins expressed in live neuronally differentiated cells were examined by fluorescent microscopy. GFP-MJD79 (a) and MJD79-GFP (b) were detected evenly throughout the cells in all and greater than $99 \%$ of transfected cells, respectively, observed 12 days after transfection. Less than $1 \%$ of MJD79-GFP transfected cells showed polyglutamine-mediated aggregate-like GFP signals within homogenous GFP signals (c), observed 12 days after transfection. Q79-GFP transfected cells formed visible aggregates (d) in all transfected cells as early as 3 days after transfection. (B) Schematic drawing of the 'cleavage-dependent cell selection system'. Live and dead cells are shown in yellow and blue, respectively, and the cell membrane and the nuclei are shown in red and green, respectively. TM-MJDQ35-G4V16 indicates the MJD protein with a 35 glutamine stretch (MJDQ35), with a Fas transmembrane domain ${ }^{15}$ (TM) fused to its N-terminus and with Gal4VP16, a strong artificial transcription factor ${ }^{14}$ (G4V16) fused to its C-terminus. GalRE-M-Zeo indicates a reporter construct containing Gal4-response elements (GalRE) and a MTV minimum promoter ${ }^{14}$ (M) upstream of the Zeocin-resistant gene (Zeo). (C) Isolation of cells with high processing activity for the MJD protein. Percentage of cells containing visible aggregates among GFP positive live cells. Cells were examined 12 days after transfection in (a), and 3, 6, 9, and 12 days after transfection in (b). The mean values of triplicate experiments are shown. S.D. are included as bars. (a) the parental PC12 cells; B16, the PC12 subclone with the highest putative MJD-cleaving activity upon initial selection with $100 \mu \mathrm{g} / \mathrm{ml}$ Zeocin; C10, the PC12 subclone with the highest putative MJD-cleaving activity upon second selection with $400 \mu \mathrm{g} / \mathrm{ml}$ Zeocin. For the cell selection, $1 \times 10^{6} \mathrm{PC} 12$ cells were transfected with $3.2 \mu \mathrm{g}$ of pCMX-TM-MJD35-Gal4VP16 together with $0.8 \mu \mathrm{g}$ of pGalRE-MTV-Zeo using TransFAST (Promega). Three days after transfection, cells were reseeded and selected with $100 \mu \mathrm{g} / \mathrm{ml}$ Zeocin (Invitrogen), and 36 colonies were isolated and analyzed. Second selection was performed with $400 \mu \mathrm{g} / \mathrm{ml}$ Zeocin. For the aggregate assay, $7.5 \times 10^{4}$ parental PC12 cells and drug-selected subclones were transfected with $1 \mu \mathrm{g}$ of pCMX-MJD79GFP in a 6-well dish. On the following day, the medium was exchanged and neuronal differentiation was induced. ${ }^{13}$ The number of cells containing visible aggregates among GFP positive live cells were counted 3, 6, 9, and 12 days after transfection. Approximately 300 transfected cells were scored. For accuracy, experiments were repeated at least four times. (D) Specific cleavage of the MJD protein in the B16 and C10 cells. The results of Western blotting analyses of cell extracts from the parental PC12 cells (b), the B16, and the C10 cells, 6 days after transfection with MJD35-GFP (a C-terminally GFP-tagged MJD35), MJD79-GFP, Htt586-23-GFP (a C-terminally GFP-tagged 586 amino acid length N-terminal huntingtin fragment containing 23 polyglutamine repeats), and Htt649-86-GFP (a Cterminally GFP-tagged 649 amino acid length $\mathrm{N}$-terminal huntingtin fragment containing 86 polyglutamine repeats) are presented. Proteins were detected using an anti-GFP antibody. Arrows indicate bands that represent cleaved proteins. $7.5 \times 10^{4}$ parental PC12, B16, and C10 cells were transfected with $1 \mu \mathrm{g}$ of pCMXMJD35-GFP, -MJD79-GFP, -Htt586-23-GFP, or -Htt649-86-GFP using TransFAST, and cells were differentiated, collected 6 days after transfection. Ten $\mu \mathrm{g}$ of cell lysate was resolved by SDS-PAGE and electroblotted onto polyvinylidene difluoride membranes, and reacted with a rabbit anti-GFP polyclonal antibody (MBL). Signals were detected using the ECL system (Amersham Pharmacia Biotech)

these subclones, only one subclone (B16) appeared to possess increased MJD processing activity. In the B16 cells, approximately $7.5 \%$ of the GFP-positive cells contained visible aggregates 12 days after MJD79-GFP transfection, and such aggregate-positive cells eventually died several days after. Using the B16 cells, we repeated the drug selection with a higher concentration of Zeocin,
$400 \mu \mathrm{g} / \mathrm{ml}$, and were able to isolate several subclones with a further enhanced activity to make visible aggregates than the B16 cells, and hence these subclones are thought to possess much higher MJD processing activity (Figure 1C, a). Among the subclones, $\mathrm{C} 10$ cells appeared to have the highest MJD processing activities. In both the B16 and C10 cells, polyglutamine-mediated aggregates increased in a 
time-dependent manner (Figure 1C, b), and approximately $24 \%$ of GFP-positive cells contained visible aggregates in the $\mathrm{C} 10$ cells 12 days after the transfection of MJD79-GFP. Compared with the parental cells, cell death was clearly observed in the B16 and $\mathrm{C} 10$ cells upon transfection with MJD79-GFP, C10 cells exhibiting stronger cell death than B16 cells (data not shown). The true percentage of aggregate-positive $\mathrm{C} 10$ cells is probably even higher, as at this time point; many GFP-positive dead cells were floating in the culture medium, which was more evident than in the B16 cells. Aggregate formation was not observed in the B16 and $\mathrm{C} 10$ cells upon transfection with MJD35-GFP, and there was no enhancement of cell death when compared with the transfection of GFP only (data not shown).

We were able to biochemically confirm the specific processing of the MJD protein in the B16 and C10 cells via Western blotting (Figure 1D). The transfected full-length MJD35-GFP and MJD79-GFP proteins were detected as $65 \mathrm{kDa}$ and $73 \mathrm{kDa}$ bands respectively, using an anti-GFP antibody. In addition to these bands, cell extracts from the B16 and C10 cells showed an additional band of approximately $40 \mathrm{kDa}$ in the MJD35-GFP transfection and approximately $46 \mathrm{kDa}$ in the MJD79-GFP transfection. The polyglutamine repeat length did not affect the efficiencies of cleavage of the MJD protein in these cells. To test the cleavage specificity of the B16 and C10 cells, we examined the ability of these cells to cleave huntingtin and the androgen receptor, gene products responsible for $\mathrm{HD}$ and SBMA, respectively. We could not detect any cleavage products of huntingtin (Figure 1D) or the androgen receptor (not shown) that were enhanced in the B16 and C10 cells. These results clearly indicated that the processing activity enhanced in both the B16 and C10 cells is specific for the MJD protein, showing that this 'cleavage-dependent cell selection system' can indeed be used for the selection of cells with enhanced cleavage activity for a particular protein.

A rough estimation of the cleavage site of the MJD protein from the sizes of the cleaved products suggests that the MJD protein is cleaved in these cells around the 250th amino acid residue, which is towards the C-terminus of the protein. $^{2}$ Our preliminary data from NMR structural analysis of bacterially synthesized MJD protein indicated that the MJD protein possesses a higher ordered structure in its $\mathrm{N}$ terminal portion and a relatively free structure around its $\mathrm{C}$ terminus, where the polyglutamine stretch resides. Consistent with this, trypsinization specifically cleaved the MJD protein at a single site after the 206th Lysine ${ }^{2}$ (Y Kamei and A Kakizuka unpublished data), which is located near the predicted cleavage region observed in the B16 and $\mathrm{C} 10$ cells. Mutation of this trypsin cleavage site in the MJD protein still allowed the protein to be cleaved in B16 and C10 cells (data not shown), indicating that the MJD processing being observed is not mediated by trypsin or trypsin-like proteases. These selected cells, especially the C10 cells will be useful for further characterization of the MJD protein processing activity and for the identification of the yet unknown MJD processing enzyme. Application of this system to other proteins will definitely open a new field in protein processing research.

\section{Y Yamamoto ${ }^{1}$, H Hasegawa ${ }^{1}$, K Tanaka $^{1}$ and A Kakizuka ${ }^{*, 1,2,3}$ \\ ${ }^{1}$ The Fourth Department, Osaka Bioscience Institute, Osaka 565-0874, Japan ${ }^{2}$ CREST, JST (Japan Science and Technology Corporation) \\ ${ }^{3}$ Current address: Graduate School of Biostudies, Kyoto University, Kyoto 606- 8501, Japan \\ * Corresponding author: A Kakizuka, Graduate School of Biostudies, Kyoto University, Kyoto 606-8501, Japan. Tel: +81-75-753-7675; Fax: +81-75-753-7676; E-mail: kakizuka@lif.kyoto-u.ac.jp}

1. Huntington's Disease Collaborative Research Group Cell (1993) 72: 971

2. Kawaguchi Y et al. Nat. Genet. (1994) 8: 221

3. La Spada AR et al. Nature (1991) 352: 77

4. Kakizuka A. Curr. Opin. Neurol. (1997) 10: 285

5. Kakizuka A. Trend. Genet. (1998) 14: 396

6. Ross CA. Neuron (1995) 15: 493

7. Ikeda H et al. Nat. Genet. (1996) 13: 196

8. DiFiglia M et al. Science (1997) 277: 1990

9. Li M et al. Ann. Neurol. (1998) 44: 249

10. Schmidt T et al. Brain Pathol. (1998) 8: 669

11. Lunkes A and Mandel JL Hum. Molec. Genet. (1998) 7: 1355

12. Schilling $G$ et al. Neuron (1999) $24: 275$

13. Yasuda $S$ et al. Genes Cells (1999) 4: 743

14. Segawa T et al. Cancer Res. (1998) 58: 2282

15. Takebayashi H et al. Cancer Res. (1996) 56: 4164 